Rev. salud pública. 10 (1):150-159, 2008

Metanálisis/Meta-Analysis

\title{
Meta-análisis sobre juego patológico 1997-2007
}

\author{
Meta-analysis of pathological gambling \\ 1997-2007
}

Yaromir Muñoz-Molina

Escuela de Administración, Universidad Eafit. Medellín, Colombia. ymunoz@eafit.edu.co

Recibido 13 Julio 2007/Enviado para Modificación 4 Enero 2008/Aceptado 23 Enero 2008

\section{RESUMEN}

Objetivo Determinar la prevalencia del juego patológico según las variables de edad y sexo e identificar los instrumentos mas usados así como los juegos asociados a dicha patología.

Método Se propone un meta-análisis de estudios publicados entre 1997 y 2007 sobre la prevalencia del juego patológico. Para ser incluidos, los artículos debían tener una muestra probabilística, indicar el instrumento de evaluación utilizado y presentar la tasa de prevalencia.

Resultados El juego patológico afecta en mayor proporción a los hombres que a las mujeres; igualmente, el porcentaje de adolescentes con ludopatía es relativamente mas elevado que el de los adultos. Las máquinas tragaperras son los juegos más frecuentemente asociados con el juego patológico.

Conclusiones El juego patológico merece más atención desde la perspectiva de la salud pública. Los estudios de prevalencia ayudan a mejorar su comprensión.

Palabras Clave: Juego de azar, jugador patológico, evaluación, tasa de prevalencia (fuente: DeCS, BIREME)

\section{ABSTRACT}

Objective Determining the prevalence of pathological gambling related to variables such as age and sex; furthermore, identifying the most current tools used for measuring it and the kind of gaming associated with this type of obsessive behavior. Method A meta-analysis of studies concerning pathological gambling published between 1997 and 2007 was carried out. Inclusion criteria for papers consisted of having a probabilistic sample, indicating the tool used for measuring it and presenting the prevalence rate.

Results It was observed that pathological gambling affects men more than women; furthermore, there are differences amongst adults and adolescents related to this type of behaviour, the latter group having the higher prevalence rate. Video lottery terminals are the most frequently occurring type of game associated with pathological gambling. 
Conclusions Pathological gambling deserves more attention by public health managers. Prevalence studies help to understand it better.

Key Words: Gambling, pathological gambling, evaluation, prevalence (source: $\mathrm{MeSH}$, $N L M)$.

$\mathrm{P}$ ese a la innegable significación económica de los juegos de suerte y de azar en cuanto a generar empleo, atraer turismo y captar recursos destinados a la salud y otras necesidades de la sociedad, algunos investigadores cuestionan su impacto negativo sobre la salud de algunos individuos (1). El juego patológico o ludopatía es la incapacidad de controlar el deseo de apostar, lo que puede generar como consecuencia la destrucción de todo el capital económico, afectivo y social de una persona (2), afectando por supuesto su entorno familiar. La amplitud del problema se refleja en que alcanza aproximadamente a cerca del 3,0 \% de la población (3), siendo para muchos investigadores un problema que se puede prevenir (4).

El meta-análisis de Stucki muestra la amplitud del problema en el ámbito internacional, pero se limita al análisis de la prevalencia en poblaciones adultas, excluyendo los jóvenes quienes, por sus elevadas tasas de juego patológico (5), que pueden ser incluso más elevadas que entre los adultos (6), constituyen una parte interesante desde la perspectiva de salud pública.

El objetivo de este trabajo es responder las siguientes a través del análisis de los estudios efectuados entre 1997 y el 2007 publicados en diversos países:

¿Cual es la prevalencia de la ludopatía en el panorama internacional en lo referente a las variables demográficas de edad y sexo?

¿Cuáles son los países que se destacan en estudiar la prevalencia de la ludopatía? ¿Cuáles son los instrumentos utilizados para identificar la ludopatía y qué tan dispares son los resultados?

¿Cuáles son los juegos de suerte y de azar asociados a la ludopatía?

Fuentes de información

Para obtener la información se efectuó una búsqueda en las bases de datos: Ebsco, Proquest, PsycINFO, Medline, Sociological Reports y Google Scholar. Como palabras de búsqueda se incluyeron las combinaciones posibles de los términos MeSH (Medical Subject Heading): gambling, pathological gambling, prevalence, evaluation. 
Se aplicaron tres filtros: "revistas universitarias", idioma: "inglés y español", período: publicaciones entre enero de 1997 y mayo de 2007. Se identificaron cerca de 179 estudios, de los cuales 46 cumplían con los criterios buscados: a) indicar el instrumento utilizado para identificar el juego patológico; b) incluir en los resultados las tasas de prevalencia encontradas en la muestra; c) tener una muestra probabilista igual o superior a 500 participantes, y d) ser accesibles.

Los estudios seleccionados fueron sometidos a análisis descriptivos de los datos estadísticos presentados para identificar frecuencias y medias estadísticas y así formar una visión global de la problemática. El tratamiento de los datos se hizo en el software SPSS 14. Los criterios de validez de los resultados fueron principalmente el instrumento utilizado para clasificar a los jugadores y la muestra probabilística. Los instrumentos utilizados para detectar la ludopatía presentan alta convergencia entre si (3-7).

Fuera de no cumplir los criterios fijados, otras razones para excluir el $74 \%$ de los estudios arrojados en la búsqueda inicial fueron: a) el foco de interés era en poblaciones muy especificas (ex-apostadores en tratamiento, prisioneros, inmigrantes ilegales), b) el interés se centrado en otro tipo de problemas de salud como desórdenes psiquiátricos, tabaquismo, alcoholismo y drogadicción, c) el interés por diversas formas de juego en relación con otras variables psicológicas.

Los principales instrumentos de medida del juego patológico

Para clasificar el grado de ludopatía se usan principalmente dos instrumentos: el Manual Diagnóstico y estadístico DSM-IV y sus adaptaciones (8) es una escala de 10 ítems. El SOGS (South Oaks Gambling Screen) (9) y su adaptación para adolescentes, el SOGS-RA (10), es una escala que contiene 20 ítems. La puntuación es la sumatoria de los ítems respondidos positivamente, así que los cortes usados en ambos casos son: entre 1 y 4 (nivel 2) equivale a jugadores en riesgo de convertirse en jugadores patológicos y 5 o más (nivel 3) equivale a la clasificación de probable jugador patológico. Ambas escalas muestran una alta convergencia de resultados cuando se comparan (11).

\section{RESULTADOS}

En la Tabla 1 se aprecia una síntesis de los estudios analizados. Éstos son generalmente de tipo descriptivo y de corte transversal. Los estudios comparativos entre dos o más países son escasos. Muchos de los estudios norteamericanos (Canadá y Estados Unidos) se realizan en Estados o Provincias sin ser de carácter nacional. 
El 71,8 \% de los estudios de prevalencia de la ludopatía fueron realizados en Canadá, Estados Unidos y Australia. Europa tiene baja participación, destacándose principalmente Suecia, Noruega, Inglaterra, Suiza y España.

\section{Demografía}

Es interesante notar que el $39 \%$ de los estudios se efectuaron entre adolescentes y estudiantes de secundaria. Al comparar las medias estadísticas entre adultos y jóvenes, se destaca que la tasa de prevalencia de ludopatía, en el nivel tres de clasificación, es más alta en los adolescentes con una media de 3,6 \% que en los adultos cuya media se sitúa en 2,2 \%. En lo que respecta a la clasificación en el nivel 2, los adolescentes tienen una media de 9,3\% frente a 5,7 \% en los adultos.

Sexo

Se constata que hay diferencias significativas al comparar los datos con respecto a la prevalencia de la ludopatía entre los hombres, cuya media es de 5,3\% frente a las mujeres, cuya media es de 1,3\%. Estos resultados son consistentes con las revisiones de estudios sobre el juego patológico efectuadas previamente $(3,6)$.

Los tipos de juego y el juego patológico

Los estudios reportan que las apuestas más apetecidas por los jugadores son el Loto, la lotería y las instantáneas, pero éstos no son los juegos de suerte y de azar mas asociados con la ludopatía. Los juegos de alta frecuencia (máquinas tragamonedas) y los de habilidad (juego de cartas) son los que se mencionan a menudo como la preferencia de los jugadores patológicos. En cuanto a los instrumentos utilizados, el 39,1 \% de los estudios usa exclusivamente el SOGS, mientras que el 32, $6 \%$ utiliza exclusivamente el DSM-IV. Ambos instrumentos se combinan en el 19, 6\% de los casos. Los instrumentos en general ofrecen resultados bastante consistentes cuando se trata de comparar dos o más de ellos en un mismo estudio, las diferencias de resultados son, según los autores, debidas a que algunos sistemas son más conservadores que otros (56), sin embargo los dos instrumentos más utilizados presentan una correlación positiva (7).

\section{DISCUSIÓN}

Conviene destacar, en primer lugar, que pocos países adelantan sistemáticamente estudios sobre la ludopatía. En segundo lugar, la prevalencia de la ludopatía, sobretodo en adultos, es relativamente estable y consistente en diferentes países independiente del instrumento utilizado (3). 
Tabla 1. Investigaciones realizadas entre 1997 y 2007 sobre la prevalencia de ludopatía

\begin{tabular}{|c|c|c|c|c|}
\hline Autores & Pais & $\begin{array}{l}\text { Muestra } \\
\text { Utiizzada }\end{array}$ & $\begin{array}{l}\text { Instrumento } \\
\text { utilizado }\end{array}$ & $\begin{array}{c}\text { Prevalencia } \\
\text { de juego } \\
\text { paidológico } \\
\text { en } \%\end{array}$ \\
\hline Huang et al. 2007 (12) & EE UU & 20739 , estudiantes & DSM-IV & 0.8 \\
\hline Ellenbogen et al. 2007 (13) & Canada & 1265 , adolescentes & DSM-IV-MR-J & 3 \\
\hline Ellenbagen, et al. 2007 (14) & Canadá & 7819 , addescentes & DSM-IV-MR J & 3,7 \\
\hline Gill et al. $2006(15)$ & Australis & 6045 , adultos & SOGS-M & 2 \\
\hline \multirow{2}{*}{ Delfabbro et al. 2005 (16) } & Australia & 926. adolescentes & DSM-IV-J & 4,4 \\
\hline & & & SOGS-RA & 2,7 \\
\hline Olason, et al. 2006 (17) & Islandia & 750 , estudiantes & DSM-IV-MR-J & 2 \\
\hline Williams et al. 2006 (18) & Canada & 585, estudiantes & CPCI & 1,4 \\
\hline Kairouz of al. 2005 (19) & Canada & 5332 , adultos & CPGI & 1,7 \\
\hline \multicolumn{5}{|l|}{ Cunningham-Wiliams et al. } \\
\hline Cox at al. 2005 (21) & Canada & 34770 , adultos & CPGI & 2 \\
\hline \multirow{2}{*}{ Wiebe y Cox, 2005 (22) } & Canada & 1000 , adultos & SOGS-R & 1,2 \\
\hline & & & SOGS & 0,9 \\
\hline Ladouceur et all. 2005 (23) & Canada & 8 842, adultos & CPGI & 0,7 \\
\hline Westermeyer ot al. 2005 (24) & EE UU & 1228 , adultos & DSM-IV & 7,6 \\
\hline Fong y Ozorio, 2005 (25) & China & 1121 , adultos & DSM-IV Chino & 1,8 \\
\hline Petry y Mallys, 2004 (26) & EE.UU & 2986 , adultos & SOGS & 1.8 \\
\hline \multirow[t]{2}{*}{ Schofieid of al. 2004 (27) } & Australia & 1029 , adultos & soos & 1,7 \\
\hline & & & SOGS & \\
\hline \multirow[t]{3}{*}{ Welte et al. 2004 (28) } & EE.UU & 2631 , adultos & DSM-IV & 3.5 \\
\hline & Nueva & & & \\
\hline & Zelanda & 7139 , & & 1,2 \\
\hline Abbott et al. 2004 (29) & y Suecia & 6452 , adultos & SOGS-R & 1 \\
\hline \multirow{2}{*}{ Hardoon et al. 2004 (30) } & Canada & 2 336, adclescentes & DSM-IV-MR-J & 4,9 \\
\hline & & & SOGS & 2,6 \\
\hline Cox et al. 2004 (31) & Canadá & 1489 , adultos & DSM IV & 1,3 \\
\hline \multirow{2}{*}{$\begin{array}{l}\text { Langhinrichsen-Rohling et al. } \\
2004(32)\end{array}$} & & & SOGS-RA & 4.6 \\
\hline & EEUU & 1735, adolescentes & MAGS-7 & 1.7 \\
\hline Engwall et ad. 2004 (33) & EE.UU & 1348 , estudiantes & SOGS & 5,2 \\
\hline Gölestam y Johansson, 2003 & Noruega & 2014 , sdultos & DSM-IV & 0.6 \\
\hline \multirow[t]{3}{*}{$\begin{array}{l}\text { Johansson y Gotestam, } 2003 \\
\text { (35) }\end{array}$} & Noruega & 3237 , addelescentes & DSM-IV & 1,7 \\
\hline & & & SOGS-RA, & 4 \\
\hline & & & DSM-IV-J, & 3,4 \\
\hline Hardoon et al. 2003 (38) & Canada & 980 . adolescentes & $\mathrm{GA}$ & 5,8 \\
\hline Villoria L. 2003 (37) & España & 1707 , universitarios & SOGS & 4,5 \\
\hline Wong y 50,2003 (38) & China & 2004 , sdultos & DSM-IV Chino & 1,8 \\
\hline Hing y Breen, 2002 (39) & Australia & 3000 , adultos & SOGS & 3 \\
\hline \multirow[t]{2}{*}{ Lupu et al. $2002(40)$} & Rumania & 500 , adolescents & $\mathrm{GA}$ & 6,8 \\
\hline & & & SOGS-RA & 1,3 \\
\hline Shapira et al. 2002 (41) & EE UU & 1051 , addescentes & DSM-IV & 3,8 \\
\hline \multirow[t]{2}{*}{ Welte et al. $2001(42)$} & EE.UU & 2638 , adultos & SOGS & 1,9 \\
\hline & & & SOGS & \\
\hline Volberg of al. 2001 (43) & Suacia & 9917 , adultos & DSM-IV & 0.6 \\
\hline \multirow[t]{3}{*}{ Poulin, $2000(44)$} & Canadá & 13549 , adolescontes & SOGS-RA & 6,4 \\
\hline & & & SOGS-RA, & 5,3 \\
\hline & & & DSM-IV-J. & 3,4 \\
\hline Derewensky y Gupta, 2000 (7) & Canadá & 980, adolescents & GA-20 & 6 \\
\hline \multirow{2}{*}{ Cox et al. $2000(45)$} & Canadá & 738, adultos & SOGS & 3,5 \\
\hline & & & & A: 1,1 y 1,8 \\
\hline Jacques et al. 2000 (a) (46) & Canada & 880, adultos & soos & B: 0.9 y 0.5 \\
\hline Bondolfi et al. $2000(47)$ & Sulza & 2526 , adultos & SOGS & 0,8 \\
\hline Ladouceur et al. 1999 (48) & Canadda & 1257, adultos & SOGS & 2,1 \\
\hline Ladouceur et el. 1999 (49) & Canada & 3426, estudiantes & soos & 2,6 \\
\hline Fisher, 1999 (50) & Inglaterra & 9774 , estudiantes & DSM-IV-MR-J & 5,6 \\
\hline Winters et al. 1998 (51) & EE.UU & 1361 , estudiantes & SOGS & 3 \\
\hline Gupta y Derevensky, 1998 (52) & Canada & 817, adolescents & DSM-IV-J & 4,7 \\
\hline Blaszczinsky ot al. 1998 (53) & Australia & 2000 , adultos & SOGS & 2,9 \\
\hline Cunningham-Wiliams et al. & & & & \\
\hline 1998 (54) & EE UU & 3004 , adultos & DSM-III & 0.9 \\
\hline Villa et al. 1997 (55) & Espaกลa & 2185 , estudiantes & DSM-IV-J & 1,6 \\
\hline
\end{tabular}

studio lengitudinal 1006 y 199 ? elechusdo en dos chudades. A: Hul y b: Quebec. en Canada. 
Cabe subrayar, sin embargo, que dicha prevalencia es relativamente más elevada entre adolescentes, lo que traza la continuidad del problema si se tiene en cuenta que muchos jugadores patológicos declaran que comienzan sus hábitos de juego a temprana edad (57). Ello implica que se requieren estudios que observen la trayectoria de los apostadores.

En tercer lugar, el problema de la ludopatía parece tener género propio. No obstante, cabe subrayar que no hay estudios longitudinales que revelen la evolución del problema para mirar más en detalle tal diferencia de sexos. En cuarto lugar, cabe resaltar que los juegos de alta frecuencia, como las máquinas tragamonedas, suelen ser más adictivos, lo cual se constata en la casi totalidad de los estudios.

Finalmente, es notoria la ausencia de estudios de prevalencia sobre el juego patológico en muchos países, sobre todo en Europa, Asia, África y Latinoamérica. Dicha ausencia puede explicarse por: falta de interés en publicarlos, existencia de otras prioridades o ausencia de consenso sobre la problemática.

Conclusiones y oportunidades de investigación

El juego patológico constituye una problemática que merece la atención desde la óptica de la salud pública. Los estudios de prevalencia reflejan el consenso sobre el problema desde dicha perspectiva. La ausencia de estudios al respecto, particularmente en la región Latinoamericana, constituye un reto y una oportunidad. Determinar la prevalencia de un problema en la población es esencial para el desarrollo de un plan de atención eficiente •

\section{REFERENCIAS}

1. Netemeyer RG, Burton S, Cole LK, Williamson DA, Zucker N, Bertman L, et al. Characteristics and beliefs associated with probable pathological gambling: a pilot study with implications for the National Gambling Impact and Policy Commission. Journal of Public Policy \& Marketing 1998; 17(2): 147-160.

2. Ratelle CF, Vallerand RJ, Mageau GA, Rousseau FL, Provencher P. When passion leads to problematic outcomes: a look at gambling. Journal of Gambling Studies 2004; 20(2): 105-119.

3. Stucki S, Rihs-Middel M. Prevalence of adult problem and pathological gambling between 2000 and 2005: An update. Journal of Gambling Studies 2007; 23(3): 245-257. 
156 REVISTADE SALUD PÚBLICA · Volumen 10 (1), Febrero 2008

4. Korn DA, Shaffer HJ. Gambling and the health of the public: adopting a public health perspective. Journal of Gambling Studies 1999; 15(4): 289-365.

5. Stinchfield R, Hanson WE, Olson DH. Problem and pathological gambling among college students. New Directions for Student Services 2006; 113: 63-72.

6. Shaffer HJ, Hall MN, Bilt JV. Estimating the prevalence of disordered gambling behavior in the United States and Canada: a research synthesis. American Journal of Public Health 1999; 89(9): 1369-1376.

7. Derevensky JL, Gupta R. Prevalence estimates of adolescent gambling: a comparison of the SOGS-RA, DSM-IV-J, and the GA 20 questions. Journal of Gambling Studies 2000; 16(2-3): 227-251.

8. American Psychiatric Association (APA). Diagnostic and Statistical Manual of Mental Disorders. 4th edition. Washington, DC: American Psychiatric Pub Inc.; 1994.

9. Lesieur HR, Blume SB. The South Oaks Gambling Screen (SOGS): a new instrument for the identification of pathological gamblers. American Journal of Psychiatry 1987; 144: 1184-1188.

10. Winters K, Stinchfield R, Fulkerson J. Patterns and characteristics of adolescent gambling. Journal of Gambling Studies 1993; 9(4): 371-386.

11. Stinchfield R. Reliability, validity, and classification accuracy of the South Oaks Gambling Screen (SOGS). Addictive Behaviors 2002; 27(1): 1-19

12. Huang JH, Jacobs DF, Derevensky JL, Gupta R, Paskus TS. Gambling and health risk behaviors among U.S. college student-athletes: findings from a national study. Journal of Adolescent Health 2007; 40: 390-397

13. Ellenbogen S, Gupta R, Derevensky JL. A cross-cultural study of gambling behaviour among adolescents. Journal of Gambling Studies 2007; 23(1): 25-39.

14. Ellenbogen S, Derevensky J, Gupta R. Gender differences among adolescents with gambling-related problems. Journal of Gambling Studies 2007; 23(2): 133-143.

15. Gill T, Grande ED, Taylor AW. Factors associated with gamblers: a population-based cross-sectional study of south Australian adults. Journal of Gambling Studies 2006; 22(2): 143-164.

16. Delfabbro PH, Lahn J, Grabosky P. Psychosocial correlates of problem gambling in Australian students. Australian and New Zealand Journal of Psychiatry 2006; 40:587-595.

17. Olason D, Sigurdardottir KJ, Smari J. Prevalence estimates of gambling participation and problem gambling among 16-18-year-old students in Iceland: a comparison of the SOGS-RA and DSM-IV-MR-J. Journal of Gambling Studies 2006; 22(1): 23-39.

18. Williams RJ, Connolly D, Wood RT, Nowatzki N. Gambling and problem gambling in a sample of university students. Journal of Gambling Issues 2006; 16: [Internet]. Disponible en: http://www.camh.net/egambling/issue16/index.html. Consultado Junio del 2007. 
19. Kairouz S, Nadeau L, LoSiou G. Area variations in the prevalence of substance use and gambling behaviours and problems in Quebec: a multilevel analysis. Canadian Journal of Psychiatry 2005; 50(10): 591-598.

20. Cunningham-Williams RM, Grucza RA, Cottler LB, Womack SB, Books SJ, Przybeck TR, et al. Prevalence and predictors of pathological gambling: results from the St. Louis personality, health and lifestyle (SLPHL) study. Journal of Psychiatric Research 2005; 39: 377-390.

21. Cox BJ, Yu N, Afifi TO, Ladouceur R. A national survey of gambling problems in Canada. Canadian Journal of Psychiatry 2005; 50(4): 213-217.

22. Wiebe JM, Cox BJ. Problem and probable pathological gambling among older adults assessed by the SOGS-R. Journal of Gambling Studies 2005; 21(2): 205-221.

23. Ladouceur R, Jacques Ch, Chevalier S, Sévigny S, Hamel D. Prevalence of Pathological Gambling in Quebec in 2002. Canadian Journal of Psychiatry 2005; 50(8): 451456.

24. Westermeyer J, Canive J, Garrard J, Thuras P, Thompson J. Lifetime prevalence of pathological gambling among american indian and hispanic american veterans. American Journal of Public Health 2005; 95(5): 860-866.

25. Fong DK, Ozorio B. Gambling participation and prevalence estimates of pathological gambling in a far-east gambling city: Macao. UNLV Gaming Research \& Review Journal 2005; 9(2): 15-28.

26. Petry N, Mallya S. Gambling participation and problems among employees at a university health center. Journal of Gambling Studies 2004; 20(2): 155-170.

27. Schofield G, Mummery K, Wang W, Dickson G. Epidemiological study of gambling in the non-metropolitan region of central Queensland. Australian Journal of Rural Health 2004; 12: 6-10.

28. Welte JW, Wieczorek WF, Barnes GM, Tidwell MC, Hoffman JH. The relationship of ecological and geographic factors to gambling behavior and pathology. Journal of Gambling Studies 2004; 20(4): 405-423.

29. Abbott MW, Volberg RA, Rönnberg S. Comparing the New Zealand and Swedish national surveys of gambling and problem gambling. Journal of Gambling Studies 2004; 20(3):237-258.

30. Hardoon KK, Gupta R, Derevensky JL. Psychosocial variables associated with adolescent gambling. Psychology of Addictive Behaviors 2004; 18(2): 170-179.

31. Cox BJ, Enns MW, Michaud V. Comparisons between the South Oaks Gambling Screen and a DSM-IV-Based interview in a community survey of problem gambling. Canadian Journal of Psychiatry 2004; 49(4): 258-264

32. Langhinrichsen-Rohling J, Rhode P, Seeley JR, Rohling ML. Individual, family, and peer correlates of adolescent gambling. Journal of Gambling Studies 2004; 20(1): 23-46. 
33. Engwall D, Hunter R, Steinberg M. Gambling and other risk behaviors on university campuses. Journal of American College Health 2004; 52(6): 245-255.

34. Götestam KG, Johansson A. Characteristics of gambling and problematic gambling in the Norwegian context: A DSM-IV-based telephone interview study. Addictive Behaviors 2003; 28(1): 189-197.

35. Johansson A, Götestam KG. Gambling and problematic gambling with money among Norwegian youth (12-18 years). Nord Journal of Psychiatry 2003; 57(4): 317-321.

36. Hardoon K, Derevensky JL, Gupta R. Empirical measures vs. perceived gambling severity among youth: why adolescent problem gamblers fail to seek treatment. Addictive Behaviors 2003; 28: 933-946.

37. Villoria C. El juego patológico en los universitarios de la comunidad de Madrid. Clínica y Salud 2003; 14(1): 43-65.

38. Wong ILK, So EMT. Prevalence estimates of problem and pathological gambling in Hong Kong. American Journal of Psychiatry 2003; 160(7): 1353-1356.

39. Hing N, Breen H. A profile of gaming machine players in clubs in Sydney, Australia. Journal of Gambling Studies 2002; 18(2): 185-205.

40. Lupu V, Onaca E, Lupu D. The prevalence of pathological gambling in Romanian teenagers. Minerva Medica 2002; 93(5): 413-418.

41. Shapira NA, Ferguson MA, Frost-Pineda K, Gold MS. Gambling and problem gambling prevalence among adolescents in Florida. [Internet]. Disponible en: http:// www.austgamingcouncil.org.au/images/pdf/eLibrary/1348.pdf. Consultado Junio del 2007

42. Welte J, Barnes G, Wieczorek W, Tidwell MC, Parker J. Alcohol and gambling pathology among U. S. adults: prevalence, demographic patterns and comorbidity. Journal of Studies on Alcohol 2001; 62(5): 706-712.

43. Volberg RA, Abbott MW, Rönnberg S, Munck IME. Prevalence and risks of pathological gambling in Sweden. Acta Psychiatrica Escandinavica 2001; 104: 250-256.

44. Poulin Ch. Problem gambling among adolescent students in the Atlantic provinces of Canada. Journal of Gambling Studies 2000; 16(1): 53-78.

45. Cox BJ, Kwong J, Michaud V, Enns MW. Problem and probable pathological gambling: considerations from a community survey. Canadian Journal of Psychiatry 2000; 45:548-553.

46. Jacques $C$, Ladouceur R, Ferland F. Impact of availability on gambling: a longitudinal study. Canadian Journal of Psychiatry 2000; 45(9): 810-815.

47. Bondolfi G, Osiek C, Ferrero F. Prevalence estimates of pathological gambling in Zwitzerland. Acta Psychiatrica Scandinavica 2000; 101: 473-475.

48. Ladouceur R, Jacques C, Ferland F, Giroux I. Prevalence of problem gambling: a replication study 7 years later. Canadian Journal of Psychiatry 1999; 44(8): 802804. 
49. Ladouceur R, Boudreault N, Jacques C, Vitaro F. Pathological gambling and related problems among adolescents. Journal of Child and Adolescent Substance Abuse 1999; 8(4): 55-68.

50. Fisher S. A prevalence study of gambling and problem gambling in British adolescents. Addiction Research 1999; 7(6): 509-538.

51. Winters KC, Bengston P, Dorr D, Stinchfield R. Prevalence and risk factors of problem gambling among college students. Psychology of Addictive Behaviors 1998; 12(2): 127-135.

52. Gupta R, Derevensky JL. Adolescent gambling behavior: a prevalence study and examination of the correlates associated with problem gambling. Journal of Gambling Studies 1998; 14(4): 319-345.

53. Blaszczynski A, Huynh S, Dumlao VJ, Farrell E. Problem gambling within a Chinese speaking community. Journal of Gambling Studies 1998; 14(4): 359-380.

54. Cunningham-Williams RM, Cottler LB, Compton III WM, Spitznagel EL. Taking chances: problem gamblers and mental health disorders - results from the St. Louis epidemiologic catchment area Study. American Journal of Public Health 1998; 88(7): 1093-1096.

55. Villa A, Becoña E, Vázquez FL. Juego patológico con máquinas tragaperras en una muestra de escolares de Gijón. Adicciones 1997; 9(2): 195-208.

56. Langhinrichsen-Rohling J, Rohling ML, Rohde P, Seeley JR. The SOGS-RA vs. the MAGS-7: prevalence estimates and classification congruence. Journal of Gambling Studies 2004; 20(3): 259-281.

57. Volberg RA. The prevalence and demographics of pathological gamblers: implications for public health. American Journal of Public Health 1994; 84(2): 237-241. 